\title{
The Supergiant Fast X-ray Transient with the shortest orbital period: Suzaku observes one orbit in IGR J16479-4514
}

\author{
Lara Sidoli* \\ INAF, Istituto di Astrofisica Spaziale e Fisica Cosmica, \\ Via E. Bassini 15, I-20133 Milano, Italy \\ E-mail: sidolidiasf-milano.inaf.it \\ P. Esposito, ${ }^{a}$ V. Sguera,${ }^{b}$ A. Bodaghee, ${ }^{c}$ J.A. Tomsick, ${ }^{c}$ K. Pottschmidt, ${ }^{d, e}$ \\ J. Rodriguez, ${ }^{f}$ P. Romano, ${ }^{g}$ J. Wilms, ${ }^{h}$ \\ ${ }^{a}$ INAF-IASF Milano, Italy \\ ${ }^{b}$ INAF-IASF Bologna, Italy \\ ${ }^{c}$ SSL, University of California, Berkeley, CA, USA \\ ${ }^{d}$ CRESST-NASA/GSFC, Greenbelt, MD, USA \\ ${ }^{e}$ CSST, University of Maryland Baltimore County, Baltimore, MD, USA \\ ${ }^{f}$ AIM - Univ. Paris VII and CEA Saclay, France \\ ${ }^{g}$ INAF-IASF Palermo, Italy \\ ${ }^{h}$ Remeis-Observatory Bamberg, Germany
}

\begin{abstract}
The eclipsing hard X-ray source IGR J16479-4514 is the Supergiant Fast X-ray Transient (SFXT) with the shortest orbital period ( $\mathrm{P}_{\text {orb }} \sim 3.32$ days). This allowed us to perform a $250 \mathrm{ks}$ long $\mathrm{X}$-ray observation with Suzaku in 2012 February, covering most of its orbit, including the eclipse egress. Outside the eclipse, the source luminosity is around a few $10^{34} \mathrm{erg} \mathrm{s}^{-1}$. The $\mathrm{X}$-ray spectrum can be fit with an absorbed power law together with a neutral iron emission line at $6.4 \mathrm{keV}$. The column density, $\mathrm{N}_{\mathrm{H}}$, is constant at $\sim 10^{23} \mathrm{~cm}^{-2}$ outside the $\mathrm{X}$-ray eclipse. During the eclipse it is lower, consistent with a scattering origin for the low $\mathrm{X}$-ray emission during the eclipse by the supergiant companion. The scattered $\mathrm{X}$-ray emission during the $\mathrm{X}$-ray eclipse is used to directly probe the density, $\rho_{\mathrm{w}}$, of the companion wind at the orbital separation, resulting in $\rho_{\mathrm{w}}=7 \times 10^{-14} \mathrm{~g} \mathrm{~cm}^{-3}$, which translates into a ratio $\dot{M}_{w} / \nu_{\infty}=7 \times 10^{-17} \mathrm{M}_{\odot} / \mathrm{km}$ of the wind mass loss rate to the wind terminal velocity. This ratio, assuming reasonable terminal velocities in the range 500-3000 $\mathrm{km} \mathrm{s}^{-1}$, translates into an accretion luminosity two orders of magnitude higher than that observed. We conclude that a mechanism reducing the accretion rate onto the compact object is at work, likely due to the neutron star magnetosphere.
\end{abstract}

An INTEGRAL view of the high-energy sky (the first 10 years) - 9th INTEGRAL Workshop and celebration of the 10th anniversary of the launch

15-19 October 2012

Bibliotheque Nationale de France, Paris, France

* Speaker. 


\section{Suzaku observes IGR J16479-4514}

IGR J16479-4514 was discovered by INTEGRAL in 2003 (Molkov et al. 2003) at hard energies $(18-50 \mathrm{keV})$, as a source displaying recurrent, transient and flaring activity reaching 600 mCrab (20-60 keV; Sguera et al. 2005, 2006). The counterpart, accurately localized with Chandra (Ratti et al. 2010), is an O-type supergiant at a distance of $2.8_{-1.7}^{+4.9} \mathrm{kpc}$ (Nespoli et al. 2008). The peculiar X-ray properties together with the supergiant companion suggested to classify the source as a member of the Supergiant Fast X-ray Transients (SFXTs). IGR J16479-4514 shows X-ray eclipses (Bozzo et al. 2008a) and is the SFXT which displays the shortest orbital period (3.3193 \pm 0.0005 days; Jain et al. 2009, Romano et al. 2009). SwiftXRT monitoring has revealed that its more usual luminosity state is at a level of $10^{33}-10^{34} \mathrm{erg} \mathrm{s}^{-1}(1-10 \mathrm{keV}), 2-3$ orders of magnitude lower than the peak of the flares (Sidoli et al. 2008).

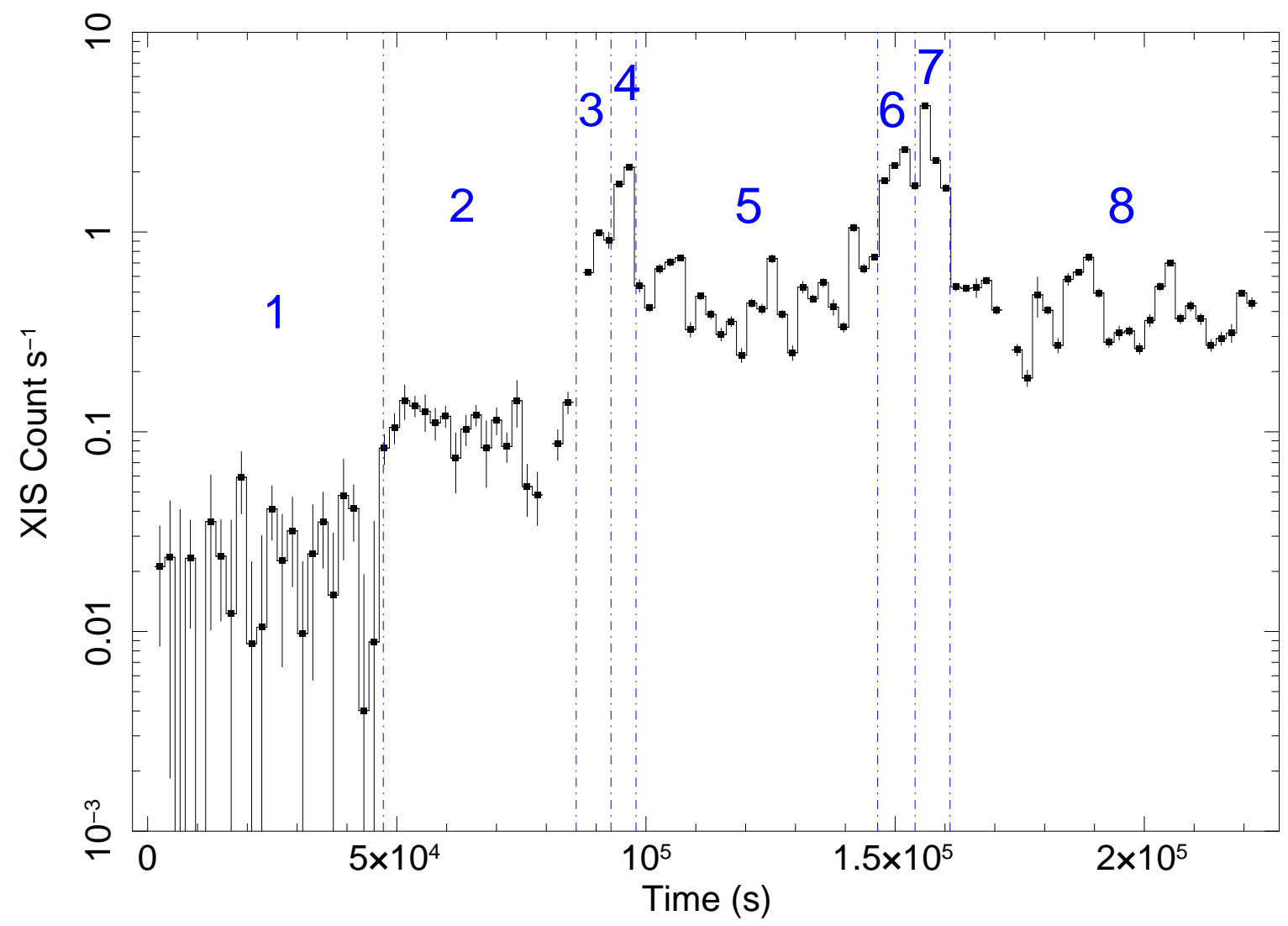

Figure 1: Suzaku/XIS (1-10 keV) light curve of IGR J16479-4514. Vertical lines and numbers indicate the time selected spectra.

We observed IGR J16479-4514 with Suzaku between 2012 February 23 and 26 (Obs ID 406078010), at the X-ray Imaging Spectrometer (XIS; Koyama et al. 2007) nominal position for a net exposure of $250 \mathrm{ks}$. The main aim of the observation was to obtain an in-depth investigation of the variability of the X-ray and supergiant wind properties along the orbit (more details about the data reduction and analysis can be found in Sidoli et al. 2013). 
The $1-10 \mathrm{keV}$ light curve is displayed in Fig. $\mathrm{W}$ and covers about $80 \%$ of the orbit. Also the eight time intervals used to perform a temporal selected spectroscopy are marked with vertical lines. The very low intensity state at the start of the observation is produced by the eclipse of the central $\mathrm{X}$-ray source by the O-type supergiant. Two faint flares, lasting 10-15 ks, are present outside the eclipse, reaching a peak flux of $3-4 \times 10^{-11} \mathrm{erg} \mathrm{cm}^{-2} \mathrm{~s}^{-1}$, spaced by $\Delta \phi=0.2$ in orbital phase. Interestingly, the first flare is located at a similar orbital phase as other bright flares observed in the past from the same source (Bozzo et al. 2009). This might suggest that a phase-locked gas structure originating from the supergiant is present along the orbit, triggering the enhanced accretion rate that produces the X-ray flare, as the compact object crosses it. Alternatively, the orbit could be mildly eccentric (although the very short orbital period favors a circular orbit) and the flare is triggered near periastron.

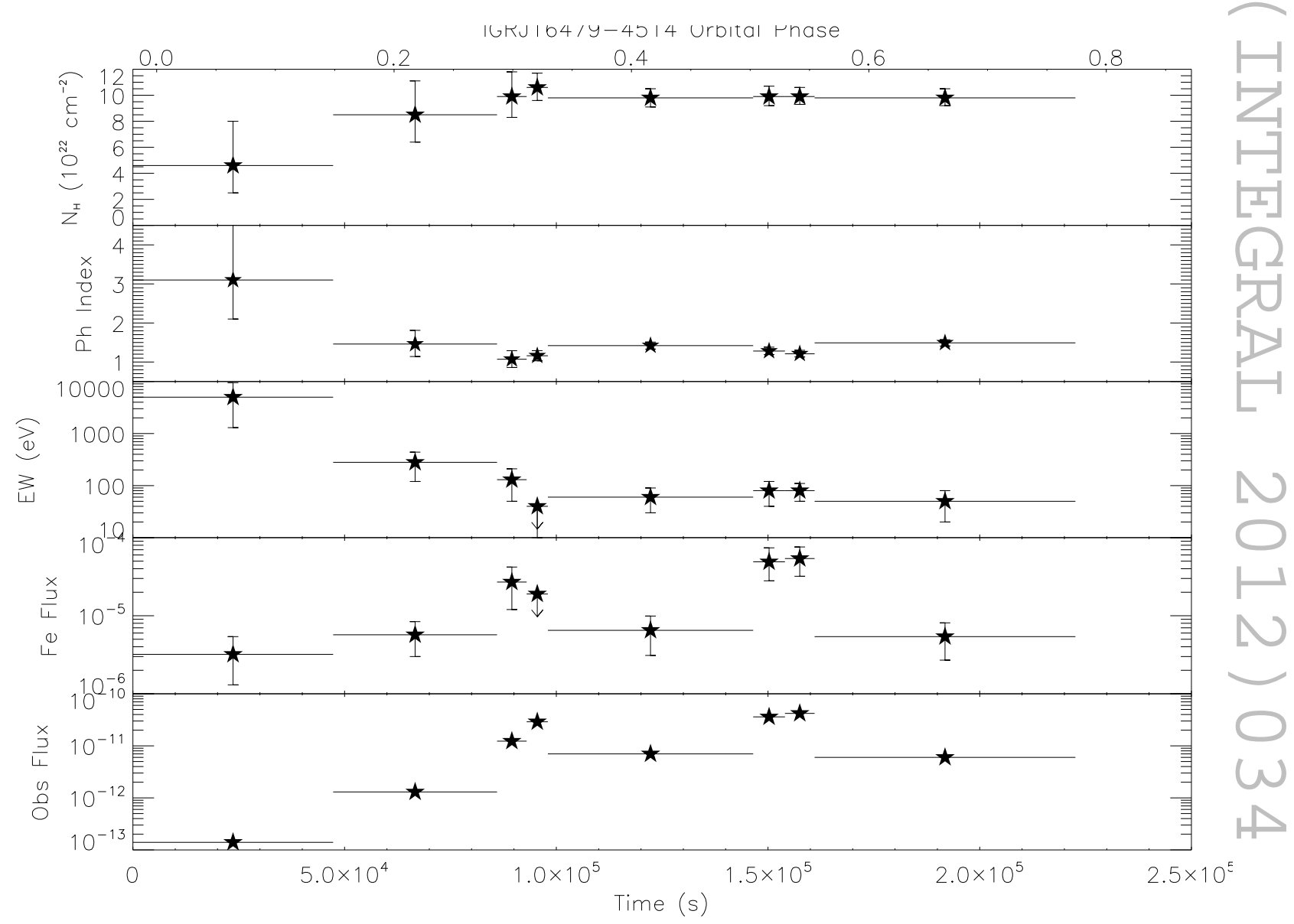

Figure 2: Suzaku/XIS (1-10 keV) temporal selected spectroscopy of IGR J16479-4514 (the eight time intervals are marked in Fig. 1). The upper $\mathrm{X}$-axis reports the orbital phase, assuming the orbital period of $286792 \mathrm{~s}$ (Romano et al. 2009) and epoch 54547.05418 MJD (Bozzo et al. 2009). Spectra have been fitted with an absorbed power law together with a narrow Gaussian line at $6.4 \mathrm{keV}$. From top to bottom, the spectral parameters are the following: absorbing column density, power law photon index, equivalent width (EW) of the neutral iron line (in eV) measured with respect to the power law continuum, flux of the iron line (in units of photons $\mathrm{cm}^{-2} \mathrm{~s}^{-1}$ ), observed flux (1-10 keV) in units of erg $\mathrm{cm}^{-2} \mathrm{~s}^{-1}$. 
The eight spectra could be well described by an absorbed power law together with a narrow Gaussian line with energy consistent with $6.4 \mathrm{keV}$, produced by fluorescence from cold iron in the supergiant wind. A summary of the spectral results can be found in Fig. $\square$.

Outside the eclipse, the $\mathrm{X}$-ray luminosity is at a level of $10^{34} \mathrm{erg} \mathrm{s}^{-1}$, assuming a distance of $2.8 \mathrm{kpc}$ (intra-flares $\mathrm{X}$-ray emission). The power law photon index is harder when the source is brighter, as in other SFXTs and HMXBs accreting pulsars.

The absorption does not show evidence for variability, within the uncertainties, except during the eclipse, where it is lower. This is consistent with the presence of Thomson scattering by electrons in the supergiant wind (Lewis et al. 1992).

The equivalent width of the $\mathrm{Fe} \mathrm{K}_{\alpha}$ emission line is around $100 \mathrm{eV}$ in the uneclipsed $\mathrm{X}$-ray emission, while it is much larger during the eclipse (this is due to the fact that it is measured with respect to the scattered $\mathrm{X}$-ray emission, the only component visible in this part of the orbit). The intensity of the iron emission line is variable along the orbit and correlates with the unabsorbed $\mathrm{X}$-ray flux above $7 \mathrm{keV}$, as expected (Inoue 1985).

\section{A mechanism reducing the accretion rate?}

The scattered X-ray emission observed during the X-ray eclipse can be used to estimate the density of the supergiant wind at the orbital separation, $a\left(a=2.2 \times 10^{12} \mathrm{~cm}\right.$ assuming a 3.32 days orbital period, a companion mass, $\mathrm{M}_{\text {opt }}$, of $35 \mathrm{M}_{\odot}$, and a neutron star of $1.4 \mathrm{M}_{\odot}$ ).

$\mathrm{X}$-rays observed during the eclipse are about $5 \%$ of the uneclisped, intra-flare, $\mathrm{X}$-ray intensity. The supergiant wind density can be estimate as $n_{\mathrm{w}}=0.05 /\left(a \sigma_{\mathrm{T}}\right)$, where $\sigma_{\mathrm{T}}$ is the Thomson cross section. This results into a wind density at the orbital separation, $\rho_{\mathrm{w}}(a)$, of $7 \times 10^{-14} \mathrm{~g} \mathrm{~cm}^{-3}$. Adopting the mass continuity equation, we can derive the ratio between the wind mass-loss rate, $\dot{M}_{w}$, and the wind terminal velocity, $\mathrm{v}_{\infty}$, as $\dot{M}_{w} / v_{\infty}=4 \pi a\left(a-R_{o p t}\right) \rho_{\mathrm{w}}(a)$, assuming a spherical outflowing wind and a velocity law for the wind velocity with $\beta=1$ (Castor et al. 1975).

The resulting ratio is $\dot{M}_{w} / v_{\infty}=7 \times 10^{-17} \mathrm{M}_{\odot} / \mathrm{km}$. If we assume reasonable wind terminal velocities in the interval 500-3000 $\mathrm{km} \mathrm{s}^{-1}$, the mass loss rate is $\dot{M}_{w}=1-7 \times 10^{-6} \mathrm{M}_{\odot} / \mathrm{yr}$.

Assuming Bondi-Hoyle accretion from the supergiant wind, the accretion rate onto the neutron star can be estimated from the relation $\dot{M}_{\text {acc }}=\left(\pi R_{a c c}^{2} / 4 \pi a^{2}\right) \cdot \dot{M}_{w}$, where $\mathrm{R}_{a c c}$ is the accretion radius. This accretion rate implies an $\mathrm{X}$-ray luminosity $\mathrm{L}_{X}=3-15 \times 10^{36} \mathrm{erg} \mathrm{s}^{-1}$, which is at least two orders of magnitude larger than observed $\left(\mathrm{L}_{\mathrm{X}} \sim 10^{34} \mathrm{erg} \mathrm{s}^{-1}\right)$.

Given that the wind density we have derived here is too high for the observed accretion luminosity, the low X-ray luminosity in IGR J16479-4514 is unlikely to be produced by direct wind accretion, implying the presence of a mechanism able to lower the accretion rate and damp the $\mathrm{X}$-ray variability implied by the structured supergiant wind. This is in agreement with the results recently obtained by Oskinova et al. (2012) in HMXBs.

A possibility is that the magnetospheric surface mediates the accretion, acting to reduce the accretion rate onto the neutron star, as already suggested by several authors to explain the peculiar X-ray behavior of the members of the SFXTs class (Bozzo et al. 2008b, Ducci et al. 2010, Shakura et al. 2012, Postnov et al. 2013, these proceedings). 


\section{Acknowledgments}

This work was supported in Italy by ASI-INAF contracts I/033/10/0 and I/009/10/0, and by the grant from PRIN-INAF 2009, "The transient X-ray sky: new classes of X-ray binaries containing neutron stars" (PI: L. Sidoli). AB received funding from NASA grant 11-ADAP11-0227.

\section{References}

[1] Bozzo, E., Stella, L., Israel, G., et al., 2008a, MNRAS, 391, L108

[2] Bozzo, E., Falanga, M., Stella, L., 2008b, ApJ, 683, 1031

[3] Bozzo, E., Giunta, A., Stella, L., 2009, A\&A, 502, 21

[4] Castor, J.I., Abbott, D.C., Klein, R.I., 1975, ApJ, 195, 157

[5] Ducci, L., Sidoli, L., Paizis, A., 2010, MNRAS, 408, 1540

[6] Inoue, H., 1985, Space Science Reviews, 40, 317

[7] Jain, C., Paul, B., Dutta, A., MNRAS, 397, L11

[8] Koyama, K., et al., 2007, PASJ, 59, 23

[9] Lewis, W., et al., 1992, ApJ, 389, 665

[10] Molkov, S., et al., 2003, The Astronomer's Telegram, 176

[11] Negueruela, I., Smith, D.M., Reig, P., et al. 2006, in ESA Spec. Pub., ed. A.Wilson, Vol. 604, 165-170

[12] Nespoli, E., Fabregat J., Mennickent, R.E., 2008, A\&A, 486, 911

[13] Oskinova, L. M., Feldmeier, A., and Kretschmar, P., 2012, MNRAS, 421, 2820

[14] Postnov, K., et al., 2013, these proceedings (arXiv:1212.2841)

[15] Ratti, E. M., et al., 2010, MNRAS, 408, 1866

[16] Romano, P., Sidoli, L., Cusumano, G. et al., 2009, MNRAS, 399, 2021

[17] Sguera, V., Barlow, E.J., Bird, A.J., et al. 2005, A\&A, 444, 221

[18] Sguera, V., Bazzano, A., Bird, A. J., et al. 2006, ApJ, 646, 452

[19] Shakura, N., Postnov, K., Kochetkova, A., Hjalmarsdotter, L., 2012, MNRAS , 420, 216

[20] Sidoli, L.; Romano, P.; Mangano, V., et a., 2008, ApJ, 687, 1230

[21] Sidoli, L., Esposito, P., Sguera, V., et al., 2013, MNRAS, in press, arXiv.1212.0723 